\title{
A TWO-DIMENSIONAL KINEMATIC MODEL OF THE LUMBRICAL IN THE HUMAN FINGER
}

\author{
J. N. A. L. Leijnse*† and J. J. Kalker \\ *Department of Plastic and Reconstructive Surgery; †Department of Biomedical Physics and Technology, \\ Erasmus University, Rotterdam, The Netherlands; and $\ddagger$ Department of Mathematics, University of \\ Technology, Delft, The Netherlands
}

\begin{abstract}
The functioning of the lumbrical muscle in the human finger is difficult to visualise. This is mainly due to the fact that the origin and insertion of the lumbrical is on tendons of other motors (the deep flexor and the extensor assembly, respectively), instead of on bone. In this paper the functioning of the lumbrical in the human finger is kinematically investigated by explicitly considering the slackness or tautness of tendon parts which are in parallel or in series with the lumbrical, and by deriving from a standard finger model equivalent representations from which the functioning of the lumbrical is visually more clear. These models are used to review and interpret the results of previous studies. Further, it is indicated that the lumbrical is in an ideal position to contribute to the control of certain fast movements, as may be of importance for the musician, and that its role in other fast movements may be limited because of its large displacements (contraction speed).
\end{abstract}

\section{NOTATION}

MCP metacarpophalangeal joint

PIP proximal interphalangeal joint

DIP distal interphalangeal joint

$P \quad$ flexor digitorum profundus

S flexor digitorum superficialis

E extensor digitorum

I interosseus

L lumbrical

M medial band of extensor, interosseus and lumbrical

$T$ lateral band of extensor, interosseus and lumbrical

$P_{p} \quad$ deep flexor tendon proximal to the insertion of the lumbrical

$P_{d} \quad$ deep flexor tendon distal to the insertion of the lumbrical

$r_{i j} \quad$ anatomic moment arms of the tendons at the resp. joints. The first index denotes the motor. The second index indicates the joint: $M C P=1, P I P=2, D I P=3$ (see Table 1).

$r_{i j}^{*} \quad$ systemic moment arm of motor $i$ at joint $j$

$\varepsilon_{\mathrm{M}}$ displacement of motor $\mathrm{M}$ (taken positive when contracting)

$0_{j} \quad$ angle of rotation of joint

$\sigma_{\mathbf{T}}$ slack in tendon $\mathrm{T}$

\section{INTRODUCTION}

In Leijnse et al. (1992), a kinematic four-tendon finger model in which the explicit quantification of the slackness of tendons allowed a concise description of the kinematically feasible and controllable free finger movements was presented. In present paper the lumbrical is implemented in this model. Anatomically, the lumbrical differs from other finger motors through its origin, which is on the tendon of another motor (the

Received in final form 26 May 1994.

Address correspondence to: J. N. A. L. Leijnse, Department of Biomedical Physics and Technology, Erasmus University, Rotterdam. The Netherlands. deep flexor) instead of on bone. As such, the lumbrical forms with the deep flexor tendon distal to its origin two parallel systemic parts which are in series with the deep flexor motor. The functioning of this system (the lumbrical-deep-flexor complex) is before all determined by the slackness or tautness of these systemic parts. In the following, these functions are kinematically investigated. Hereby the case with all tendon parts taut, which assumably represents the prime mode of lumbrical functioning, is studied in detail. While the results provide a formal mathematical analysis, the discussion presents a qualitative view based on equivalent finger model representations from which the function of the lumbrical is visually clear. Apart from the analysis of the kinematic properties of the model, central questions investigated are (i) the equivalence of the interosseus and lumbrical in the control of the finger (Thomas et al., 1968; Long, 1968), (ii) the differences in their displacements in a number of well-defined finger movements, (iii) the effect of the lumbrical at the MCP joint, as illustrative to the experiments of Backhouse and Catton (1954), Ranney et al. (1987) and Ranney and Wells (1988), (iv) the fast execution of movements with isometric lumbrical or isometric MCP.

The present study provides a quantitative kinematic synthesis of past research on lumbrical function, and presents a simple but comprehensive model for the analysis of the kinematics, or the kinematic impairments of the finger in the sagittal plane.

\section{MATERIALS AND METHODS}

\section{The kinematic finger model with lumbrical}

In this paper, the two-dimensional kinematic finger model of Leijnse et al. (1992) :

$$
\mathrm{d} \varepsilon_{\mathrm{P}}=r_{\mathrm{P} 1} \mathrm{~d} \theta_{1}+r_{\mathrm{P} 2} \mathrm{~d} \theta_{2}+r_{\mathrm{P} 3} \mathrm{~d} \theta_{3}-\mathrm{d} \sigma_{\mathrm{P}},
$$




$$
\begin{aligned}
& \mathrm{d} \varepsilon_{\mathrm{S}}=r_{\mathrm{S} 1} \mathrm{~d} \theta_{1}+r_{\mathrm{S} 2} \mathrm{~d} \theta_{2}-\mathrm{d} \sigma_{\mathrm{S}}, \\
& \mathrm{d} \varepsilon_{\mathrm{E}}=-r_{\mathrm{E} 1} \mathrm{~d} \theta_{1}-r_{\mathrm{M} 2} \mathrm{~d} \theta_{2}-\mathrm{d} \sigma_{\mathrm{E}}, \\
& \mathrm{d} \varepsilon_{\mathrm{l}}=r_{\mathrm{I} 1} \mathrm{~d} \theta_{1}-r_{\mathrm{M} 2} \mathrm{~d} \theta_{2}-\mathrm{d} \sigma_{\mathrm{I}}, \\
& \mathrm{d} \theta_{3}=\frac{r_{\mathrm{M} 2}-r_{\mathrm{T} 2}}{r_{\mathrm{T} 3}} \mathrm{~d} \theta_{2}-\frac{\mathrm{d} \sigma_{\mathrm{T}}}{r_{\mathrm{T} 3}}, \\
& \sigma_{\mathrm{E}}+\sigma_{1}+\left(\sigma_{\mathrm{P}}+\sigma_{\mathrm{T}}\right) \sigma_{\mathrm{S}}=0,
\end{aligned}
$$

is expanded with the lumbrical muscle (Fig. 1). The model (1) provides a kinematic description of the movements feasible in the free moving finger in the sagittal plane. The equations describe the changes of length ( $\mathrm{d} \varepsilon_{i}$ ) of the motors $M_{i}$ with the joint rotations $\left(\mathrm{d} \theta_{j}\right)$. The joint rotations and moment arms are taken positive for flexion. Motor contraction (shortening) is taken positive. The extensor (E), interosseus (I) and lumbrical (L) all join two common end tendons, called the medial (M) and lateral (T) band, which insert in the middle and end phalanx, respectively. Tendons can be slack; this is quantified by the non-negative slack variables $\sigma_{i}\left(\sigma_{i} \geqslant 0\right)$. The control equation (1f) states which slacks must be zero for controllable free movement. The parts of the deep flexor (P) proximal and distal to the origin of the lumbrical are further called $P_{p}$ and $P_{d}$, respectively. In the following, distinction is made between 'anatomic' and 'systemic' moment arms. Both moment arms are formally defined by the expression:

$$
r_{X_{j}}=\frac{\partial \varepsilon_{X}}{\partial \theta_{j}}
$$

With the anatomic moment arms, in expression (2) the displacements $\varepsilon_{X}$ are of the tendons $X$ when the joints are considered disjunct parts of the system, and correspond to the anatomic distance between the tendon and the joint axis of rotation. With the systemic moment arms, the displacements are the changes of motor length in well-defined conditions of systemic functioning (e.g. tautness of tendons). When the systcmic moment arm differs from the anatomic moment arm, it is written with an asterisk $\left(r_{X_{i}}^{*}\right)$.

The system equations of the lumbrical are derived as follows. The proximal part of the deep flexor (P) and the lumbrical $(\mathrm{L})$ are two motors in series. The proximal and distal part of the deep flexor $\left(\mathbf{P}_{\mathbf{p}}, \mathbf{P}_{\mathbf{d}}\right)$ are a motor and a fixed tendon length in series. The

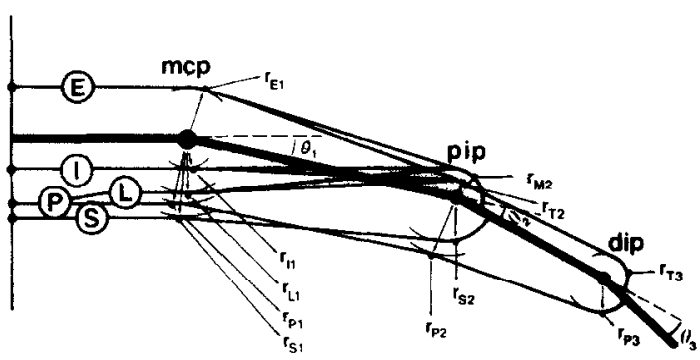

Fig. 1. Model of the finger. displacement relationships of both system parts are

$$
\begin{aligned}
\mathrm{d} \varepsilon_{\mathrm{P}}+\mathrm{d} \varepsilon_{\mathrm{L}}= & r_{\mathrm{L} .1} \mathrm{~d} \theta_{1}-r_{\mathrm{M} 2} \mathrm{~d} \theta_{2}-\mathrm{d} \sigma_{\mathrm{P}_{\mathrm{p}}}-\mathrm{d} \sigma_{\mathrm{L}} . \\
\mathrm{d} \varepsilon_{\mathrm{P}}= & r_{\mathrm{P} 1} \mathrm{~d} \theta_{1}+r_{\mathrm{P} 2} \mathrm{~d} \theta_{2}+r_{\mathrm{P} 3} \mathrm{~d} \theta_{3} \\
& -\mathrm{d} \sigma_{\mathrm{P}_{\mathrm{P}}}-\mathrm{d} \sigma_{\mathrm{P}_{\mathrm{d}}} .
\end{aligned}
$$

[Note that in equation ( $3 a$ ) the displacement of the lumbrical at the PIP is equal to the displacements of extensor and interosseus in the model (1) (same insertions), and that this displacement is determined only by the medial band (Leijnse et al., 1992).] The subtraction of expression ( $3 b$ ) from expression (3.a), and the substitution the coupling mechanism of the PIP and DIP joint [equation (le)] in the result and also in the expression (3b), results in the equations (4a) and (4e) of the displacement model of the finger with lumbrical:

$$
\begin{aligned}
\mathrm{d} \varepsilon_{\mathrm{P}} & =r_{\mathrm{P} 1} \mathrm{~d} \theta_{1}+r_{\mathrm{P} 2}^{*} \mathrm{~d} \theta_{2}-\mathrm{d} \sigma_{\mathrm{P}}^{*}, \\
\mathrm{~d} \varepsilon_{\mathrm{S}} & =r_{\mathrm{S} 1} \mathrm{~d} \theta_{1}+r_{\mathrm{S} 2} \mathrm{~d} \theta_{2}-\mathrm{d} \sigma_{\mathrm{S}}, \\
\mathrm{d} \varepsilon_{\mathrm{E}} & =-r_{\mathrm{E} 1} \mathrm{~d} \theta_{1}-r_{\mathrm{M} 2} \mathrm{~d} \theta_{2}-\mathrm{d} \sigma_{\mathrm{E}}, \\
\mathrm{d} \varepsilon_{\mathrm{I}} & =r_{\mathrm{I}} \mathrm{d} \theta_{1}-r_{\mathrm{M} 2} \mathrm{~d} \theta_{2}-\mathrm{d} \sigma_{1}, \\
\mathrm{~d} \varepsilon_{\mathrm{L}} & =-r_{\mathrm{L} 1}^{*} \mathrm{~d} \theta_{1}-r_{\mathrm{L} 2}^{*} \mathrm{~d} \theta_{2}-\mathrm{d} \sigma_{\mathrm{L}}^{*}+\mathrm{d} \sigma_{\mathrm{P}}^{*}, \\
\mathrm{~d} \theta_{3} & =\frac{r_{\mathrm{M} 2}-r_{\mathrm{T} 2} \mathrm{~d} \theta_{2}-\frac{\mathrm{d} \sigma_{\mathrm{T}}}{r_{\mathrm{T} 3}},}{r_{\mathrm{T} 3}} \\
\sigma_{\mathrm{E}} & +\sigma_{\mathrm{P}}^{*} \sigma_{\mathrm{S}}+\sigma_{\mathrm{L}}^{*} \sigma_{\mathrm{I}}=0, \\
\sigma_{i} & =\sigma_{i 0}+\int \mathrm{d} \sigma_{i} \geqslant 0 \quad \forall i,
\end{aligned}
$$

in which the systemic moment arms $r_{\mathrm{L} 1}^{*}, r_{\mathrm{L} 2}^{*}, r_{\mathrm{P} 2}^{*}$ are given by

$$
\begin{gathered}
r_{\mathrm{P} 2}^{*}=r_{\mathrm{P} 2}+\frac{r_{P 3}}{r_{\mathrm{T} 3}}\left(r_{\mathrm{M} 2}-r_{\mathrm{T} 2}\right), \\
r_{\mathrm{L} 1}^{*}=r_{\mathrm{P} 1}-r_{\mathrm{L} 1}, \\
r_{\mathrm{L} 2}^{*}=r_{\mathrm{M} 2}+r_{\mathrm{P} 2}^{*},
\end{gathered}
$$

and the generalised slack variables $\sigma_{\mathrm{P}_{\mathrm{d}} \mathrm{T}}^{*}, \sigma_{\mathrm{P}}^{*}$ and $\sigma_{\mathrm{L}}^{*}$ by

$$
\begin{gathered}
\sigma_{\mathbf{P}_{\mathrm{d}} \mathrm{T}}^{*}=\sigma_{\mathrm{P}_{\mathrm{d}}}+\left[r_{\mathrm{P} 3} / r_{\mathrm{T} 3}\right] \sigma_{\mathrm{T}}, \\
\sigma_{\mathrm{P}}^{*}=\sigma_{\mathrm{P}_{\mathrm{p}}}+\sigma_{\mathrm{P}_{\mathrm{d}} \mathrm{T}}^{*}, \\
\sigma_{\mathrm{L}}^{*}=\sigma_{\mathrm{P}_{\mathrm{p}}}+\sigma_{\mathrm{L}} .
\end{gathered}
$$

The meaning of these slacks is explained further.

\section{Anatomic data}

-In the normal finger, the moment arm of the lumbrical at the MCP is generally smaller, but almost equal to the moment arm of the deep flexor (Brand, 1985). From this it follows that

$$
r_{\mathrm{L} 1}^{*}=r_{\mathrm{P}_{1}}-r_{\mathrm{L} 1} \geqslant 0 \text {. }
$$

-During PIP flexion, the lateral band (T) at the PIP shifts palmarly and may even pass to the flexion side of the PIP joint axis (Garcia-Elias et al., 1991). This change in the moment arm $r_{T_{2}}$ determines the relative rate of DIP and PIP rotation in the coupling mechanism of expression (4f). In the stick diagrams of Fig. 4, an acceptable visual result was obtained by 
Table 1. Moment arms of the tendons

\begin{tabular}{lccccc}
\hline MCP & $r_{\mathrm{P} 1}$ & $r_{\mathrm{S} 1}$ & $r_{\mathrm{E} 1}$ & $r_{11}$ & $r_{\mathrm{L} 1}$ \\
& 11 & 13 & 9 & 6 & 9 \\
PIP & $r_{\mathrm{P} 2}$ & $r_{\mathrm{S} 2}$ & $r_{\mathrm{M} 2}$ & $r_{\mathrm{T} 2}$ & \\
& 10.5 & 8.5 & 5 & $*$ & \\
DIP & $r_{\mathrm{P} 3}$ & $r_{\mathrm{Y} 3}$ & & & \\
& 6 & 4 & & &
\end{tabular}

Note. Real values (in $\mathrm{mm}$ ) used to calculate the results. From: Spoor (1983).

${ }^{*}$ Function of PIP position, see expression (9).

approximating the moment $\operatorname{arm} r_{\mathrm{T} 2}$ as a second order polynomial of the PIP rotation $\theta_{2}$, with the following properties:

$$
\begin{aligned}
r_{\mathrm{T} 2}=r_{\mathrm{M} 2} ; & \theta_{2}=0 \\
r_{T 2}=0 ; & \theta_{2}=\frac{\pi}{2}, \\
\frac{\mathrm{d} r_{\mathrm{T} 2}}{\mathrm{~d} \theta_{2}}=0 ; & \theta_{2}=\frac{\pi}{2} .
\end{aligned}
$$

This leads to

$$
r_{\mathrm{T} 2}=r_{\mathrm{M} 2}\left[1-\frac{2 \theta_{2}}{(\pi / 2)}+\frac{\theta_{2}^{2}}{(\pi / 2)^{2}}\right] .
$$

All other moment arms are taken constant; the values used for calculation are given in Table 1.

\section{RESULTS}

Motor function as expressed by the slack variables

In this section the functioning of the model as resulting from slackness or tautness of tendon parts is analysed. First the physical meaning of the generalised slack variables is investigated.

The generalised slack variables [expression (6)]. (i) $\sigma_{P_{b} T}^{*}$ : expresses that slack in the $P_{d}$ and the lateral band $T$ can be exchanged by rotating the DIP (by external action) without altering the position of the PIP. The $P_{d}$ is therefore only truly taut, in the sense that its tautness fixes the DIP joint, when the lateral band is also taut. Only then the $P_{d}$ is a functional system element in series with the $P_{p}$

(ii) $\sigma_{\mathrm{P}}^{*}=\sigma_{\mathrm{P}_{\mathrm{p}}}+\sigma_{\mathrm{P}_{\mathrm{d}} \mathrm{T}}^{*}$ : expresses the slackness of the deep flexor, as consisting of $P_{p}$ and $P_{d}$ in series.

(iii) $\sigma_{\mathrm{L}}^{*}=\sigma_{\mathrm{P}_{\mathrm{p}}}+\sigma_{\mathrm{L}}$ : expresses the slackness of the lumbrical and $P_{p}$ in series.

The functioning of the lumbrical-deep-flexor complex as determined by the slack variables. Expression (4e) describes the change of length of the loop formed by the lumbrical $L$ and the distal part of the deep flexor $P_{d}$ in parallel. It contains a sum of slacks of opposite sign, which means that together these slacks can increase indefinitely without any effect on joint position or lumbrical motor length. Physically this corresponds to a displacement of the common origin of lumbrical and $\mathbf{P}_{d}$, which changes the slack in both parallel parts with equal quantity. The slacks themselves are the sum of a common series and an individual parallel term (expression (6)). This expresses that equal amounts of slack in both parallel parts $\left(\sigma_{\mathrm{L}}, \sigma_{\mathrm{P}_{\mathrm{d}} \mathrm{T}}^{*}\right)$ can be exchanged for an equal amount of slack in the common tendon $\left(\sigma_{\mathrm{Pp}}\right)$. The slacks distinguish following systemic functions of the lumbrical-deep-flexor complex:

(i) $\sigma_{\mathrm{L}}^{*}, \sigma_{\mathrm{P}}^{*} \neq 0$ : deep flexor inactive (e.g. when $\mathrm{P}_{\mathrm{p}}$ is cut); no function at all.

(ii) $\sigma_{\mathrm{L}}^{*} \neq 0, \sigma_{\mathrm{P}}^{*}=0$ : lumbrical inactive and deep flexor in series with its end tendon: model (1) holds.

(iii) $\sigma_{\mathrm{L}}^{*}=0, \sigma_{\mathrm{P}}^{*} \neq 0$ : lumbrical in series with the deep flexor as a digastric motor (further called $P_{L}$ ). Equation (4e) is inactive (the parallel loop of lumbrical and $P_{d}$ is slack), and the equation (3a) holds (with zero slacks). $P_{L}$ effectively functions as an interosseus, with a somewhat greater moment arm for the MCP (Long, 1968). For a clinical application, see Parkes (1970). (iv) $\sigma_{\mathrm{L}}^{*}, \sigma_{\mathrm{P}}^{*}=0$ : lumbrical and $\mathrm{P}_{\mathrm{d}}$ taut in parallel, and together in series with the taut $P_{p}$ tendon. This is assumed to be the main mode of functioning of the L-P complex, and is further investigated.

The different motor combinations which allow controlled finger movement. Equations (4) show that the displacements of the finger motors with zero slacks are only function of the proximal two joints (MCP, PIP), which means that the free moving model effectively functions as a bi-articular chain. In this chain, five motors are present, while for full control mathematically three suffice (Spoor and Landsmeer, 1976). Therefore, two motors will be mathematically redundant. In the normal finger the redundancies are with the flexors ( $P$ and $S$ ), and the intrinsics ( $L$ and $I$ ). The conditions for flexor redundancy were investigated in Spoor and Landsmeer (1976). The redundancy of the intrinsics is investigated further. These redundancies are expressed in the control equation $(4 \mathrm{~g})$, which defines the motor combinations which in the normal finger allow controlled finger movement. With the normal redundancy conditions satisfied, these motor combinations are:

(i) $\sigma_{\mathrm{P}}^{*}=0$ : the $\{\mathrm{P}, \mathrm{E}, \mathrm{I}$ or $\mathrm{L}\}$ control system. When $\sigma_{\mathrm{P}}^{*}=0$, the finger can be completely controlled by the motors E, P, I and/or L, i.e. the equations (4a) and (4c)-(4e). These equations are basically identical to the bi-articular model with lumbrical of Thomas et al., (1968).

(ii) $\sigma_{\mathrm{P}}^{*} \neq 0, \sigma_{\mathrm{S}}=0$ : the $\left\{\mathrm{S}, \mathrm{E}, \mathrm{I}\right.$ or $\left.\mathrm{P}_{\mathrm{L}}\right\}$ control system. (a) $\sigma_{\mathrm{L}}^{*} \neq 0$ : With the deep flexor and lumbrical slack, the proximal bi-articular chain of the finger can be controlled by the motors S, E, I, while the DIP remains uncontrolled [equations (4a) and (4e) are inactive].

(b) $\sigma_{L}^{*}=0$ : the $P_{L}$, i.e. the lumbrical in series with the deep flexor with the $P_{d}$ slack, may in principle also provide the interosseus function required for the control of the proximal bi-articular chain, resulting in the control system: E, S, $P_{L}$ (Long, 1968). 
The lumbrical-deep-flexor complex with zero slacks

The bi-articular equivalent model of the finger with taut tendons. In equation (4), with zero slacks, the lumbrical is mathematically indistinguishable from a motor with fixed origin. This indicates that it must be possible to represent the model (4) as a biarticular chain in which the lumbrical originates from the fixed environment, and not from the tendon of the deep flexor. This representation is given in the Fig. 2(a), and is further called the bi-articular equivalent model. Fig. 2(a) represents the lumbrical displacements as seen by an observer who sits on the tendon of the deep flexor tendon at the lumbrical origin, and moves with it. This observer notices only displacements at the distal end of the lumbrical, and can explain these displacements as a function of the MCP and the coupled PIP-DIP rotations only by assuming the systemic moment arms as present in the Fig. 2(a).

The systemic moment arms of the lumbrical-deepflexor complex with taut tendons $\left(\sigma_{\mathrm{L}}^{*}, \sigma_{\mathrm{p}}^{*}=0\right)$. With respect to the systemic moment arms of the $\mathrm{L}-\mathrm{P}$ complex, the following statements hold (Fig. 2):

(1) $r_{\mathrm{L} 1}^{*}$ : the lumbrical systemic moment arm at the MCP is of extension, and it is very small [expression

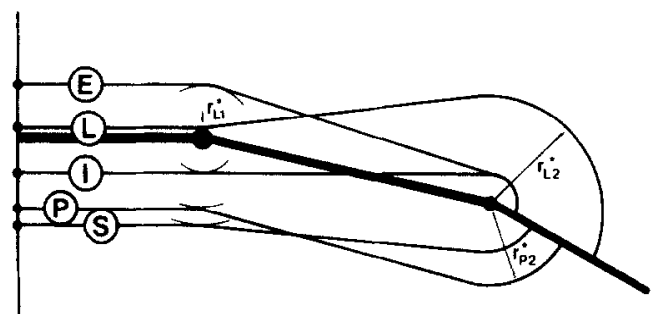

(a)

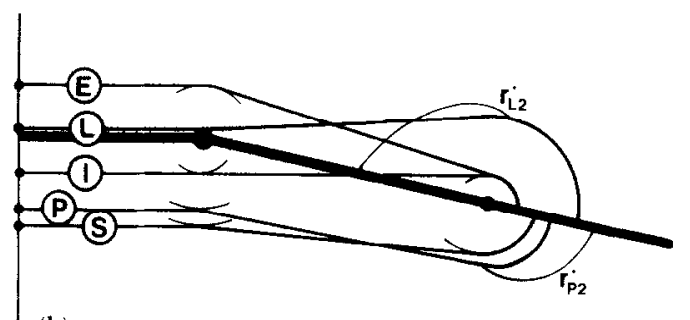

(b)

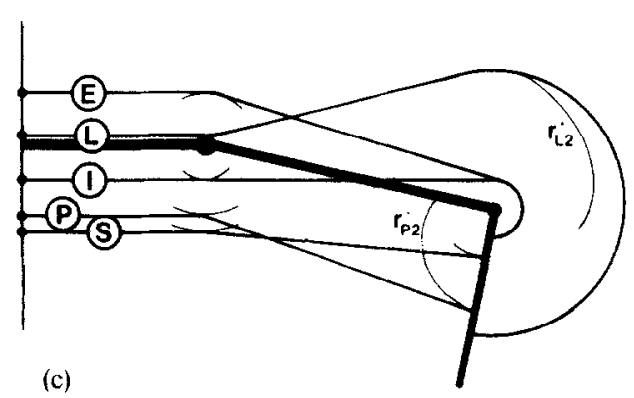

Fig. 2. (a) Bi-articular equivalent finger model; (b) systemic moment arms of lumbrical and deep flexor in extension; (c) systemic moment arms of lumbrical and deep flexor in flexion.
(7)]. When it is zero $\left(r_{\mathrm{L} 1}^{*}=0\right)$, the lumbrical length is independent of the MCP position (Stack. 1962; Thomas et al. 1968). This means that the lumbrical is effectively a quasi mono-articular finger motor of which the length is almost completely determined by the PIP position. This in contrast with the other finger motors E,P,S,I which are basically bi-articular.

(2) $r_{L 2}^{*}, r_{P 2}^{*}$ : the lumbrical (Thomas et al., 1968) and (to a lesser extent) also the deep flexor (Spoor and Landsmeer, 1976) have huge systemic moment arms $\left(r_{\mathbf{L} 2}^{*}, r_{\mathrm{P} 2}^{*}\right)$ at the PIP joint. These moment arms increase with PIP flexion [fig. 3(a)], because they depend on the variable moment arm $r_{\mathrm{T} 2}$ [from equations (5a), (5c) and (9)]. This means that displacements of the lumbrical and deep flexor vary nonlinearly with PIP rotation [Fig. 3(b)]. In Fig. 2(b) and 2(c) these systemic moment arms are visualised for the extended and the flexed finger, as calculated from expression (9). For the sake of completeness, it may be noted that with finger extension $\left(\theta_{2} \rightarrow 0\right)$. the ratio $r_{\mathrm{L} 2}^{*} / r_{\mathrm{P} 2}^{*}$ increases [Fig. 3(c)], meaning that when $\theta_{2} \rightarrow 0$ the lumbrical extension action at the PIP becomes slightly more efficient relative to the $\mathrm{P}_{\mathrm{d}}$ flexion action.

The mutual redundancy of lumbrical and interosseus in the control of the finger in the sagittal plane. In this section, the conditions for mutual redundancy of the interosseus and the lumbrical in the $\mathbf{L}-\mathrm{P}$ complex with all tendon parts taut $\left(\sigma_{\mathrm{L}}^{*}, \sigma_{\mathrm{P}}^{*}=0\right)$ are derived. Assume the finger in a non-specified position with all tendon parts taut, and replace the lumbrical by a taut and inextensible rope. In this position, keep the motors of either the motor triplet E,P,I or the motor triplet E, P, L isometric. It then holds that the finger is controllable by such a motor triplet when, while keeping the motors isometric, it is not possible to rotate (by external action) any joint. Under the above conditions only joint rotations are feasible which cause slack in tendons, as the tendons are inextensible and initially taut. The feasibility of such rotations would indicate that the slackened motors can contract without causing elongation of any other motor. This means that the resulting movement has no antagonists and that it is irreversible, and therefore that the finger cannot be properly controlled. To check this, the set of equations

$$
\begin{array}{r}
r_{\mathrm{P} 1} \mathrm{~d} \theta_{1}+r_{\mathrm{P} 2}^{*} \mathrm{~d} \theta_{2}-\mathrm{d} \sigma_{\mathrm{p}}=0, \\
-r_{\mathrm{E} 1} \mathrm{~d} \theta_{1}-r_{\mathrm{M} 2} \mathrm{~d} \theta_{2}-\mathrm{d} \sigma_{\mathrm{E}}=0, \\
r_{X 1}^{*} \mathrm{~d} \theta_{1}+r_{X 2}^{*} \mathrm{~d} \theta_{2}-\mathrm{d} \sigma_{X}=0,
\end{array}
$$

is solved for $\mathrm{d} \theta_{j}$, with the condition that the slacks must be positive: $\mathrm{d} \sigma_{\mathrm{p}}, \mathrm{d} \sigma_{\mathbf{E}}, \mathrm{d} \sigma_{\mathbf{X}} \geqslant 0$ ( $X$ stands for either $\mathrm{L}$ or I). This set has a non-trivial solution when the determinant is zero, i.e. when

$$
\begin{array}{r}
\left(r_{\mathrm{P} 1} r_{X 2}^{*}-r_{\mathrm{P} 2}^{*} r_{X 1}^{*}\right) \mathrm{d} \sigma_{\mathrm{E}}+\left(r_{X 1}^{*} r_{\mathrm{M} 2}-r_{X 2}^{*} r_{\mathrm{E} 1}\right) \mathrm{d} \sigma_{\mathrm{p}} \\
+\left(r_{\mathrm{E} 1} r_{\mathrm{P} 2}^{*}-r_{\mathrm{M} 2} r_{\mathrm{P} 1}\right) \mathrm{d} \sigma_{X}=0 .
\end{array}
$$

When in the expression (11) the coefficients of the slacks are strictly positive, the slacks must be zero ( $\left.\mathrm{d} \sigma_{i}=0\right)$, and the rank of the moment arm matrix in 

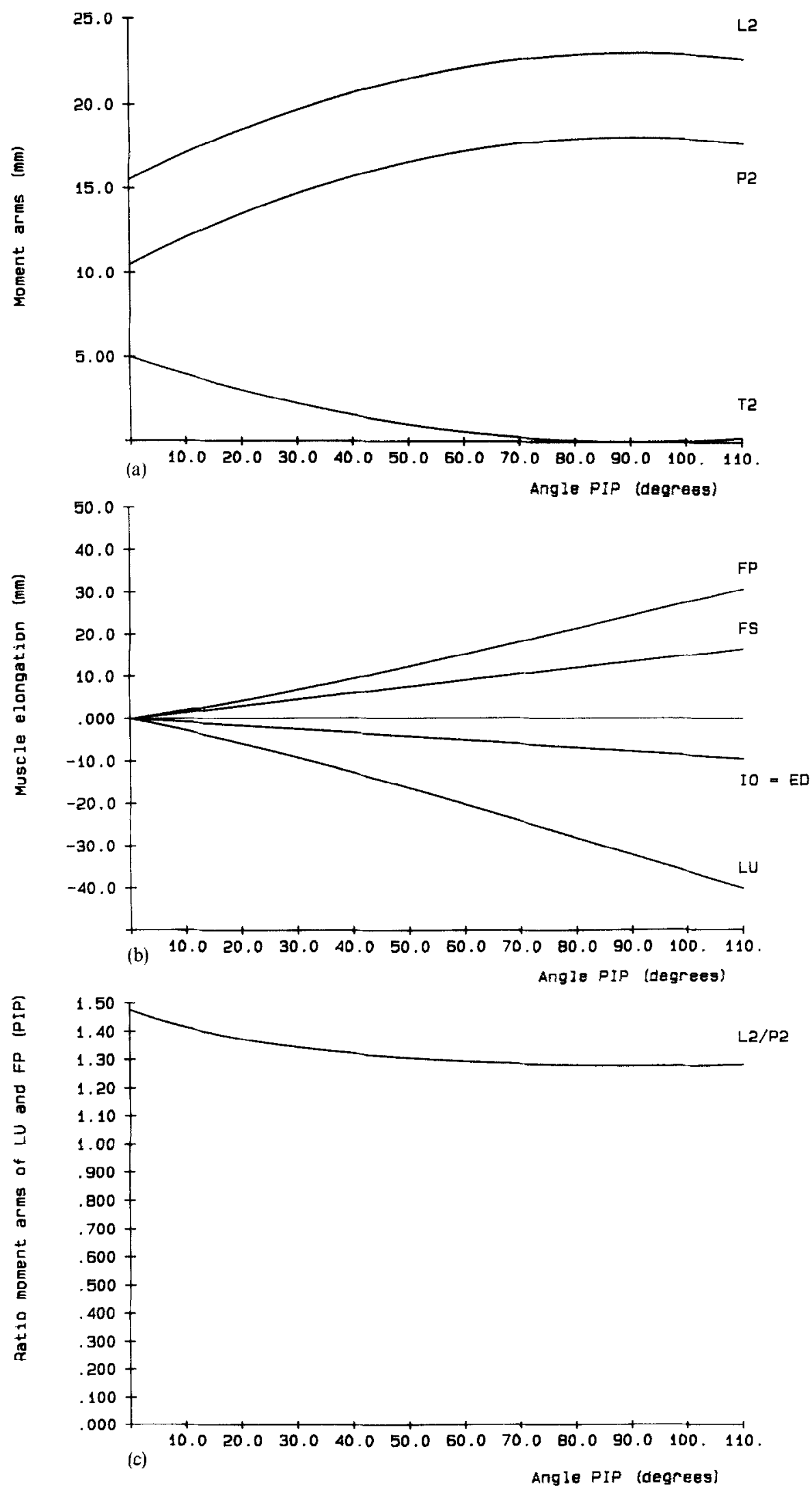

Fig. 3. Systemic moment arms as calculated from Table 1 and expression (9): (a) systemic moment arms as 1 function of the PIP position. T: lateral band, P: deep flexor, L: lumbrical; (b) muscle displacements as a function of the PIP position; (c) ratio of the systemic moment arms $r_{\mathrm{L} 2}^{*} / r_{\mathrm{P} 2}^{*}$ as a function of the PIP position. 
expression (10) is equal to two. Expression (10) then only allows the zero solution $\left(\mathrm{d} \theta_{i}=0\right)$, meaning that no joint can be moved without elongation of tendons, and that the chain is controllable. The positivity of the slack coefficients leads to the following conditions:

$$
\begin{aligned}
& \frac{r_{\mathrm{E} 1}}{r_{\mathrm{P} 1}}-\frac{r_{\mathrm{M} 2}}{r_{\mathrm{P} 2}^{*}}>0 \\
& \frac{r_{\mathrm{I} 1}}{r_{\mathrm{E} 1}}+\frac{r_{\mathrm{M} 2}}{r_{\mathrm{M} 2}}>0
\end{aligned}
$$
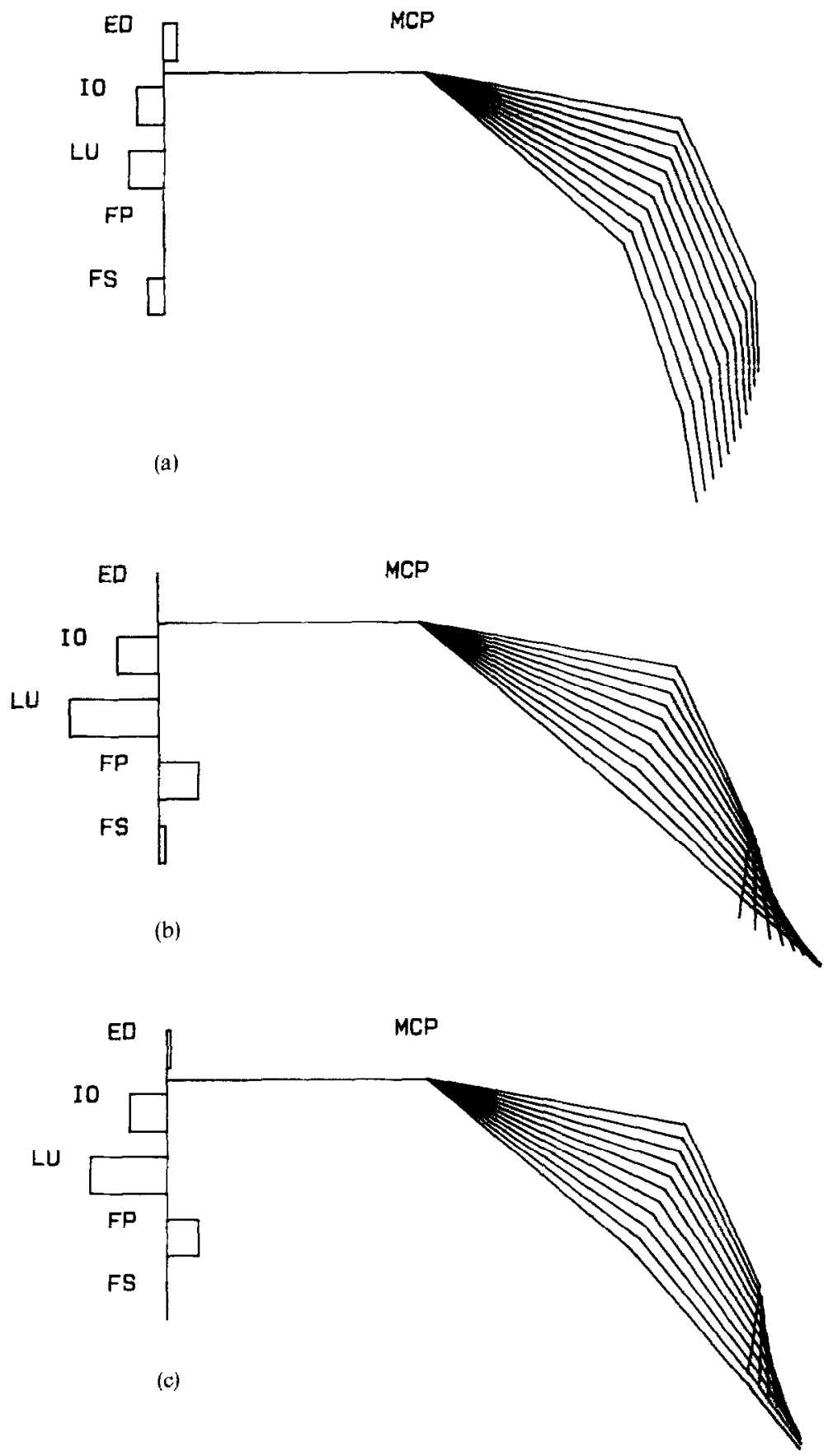

Fig. $4(a-c)$.

$$
\begin{aligned}
& \frac{r_{\mathrm{I} 1}}{r_{\mathrm{P} 1}}+\frac{r_{\mathrm{M} 2}}{r_{\mathrm{P} 2}^{*}}>0 \\
& \frac{r_{\mathrm{L} 2}^{*}}{r_{\mathrm{M} 2}}-\frac{r_{\mathrm{L} 1}^{*}}{r_{\mathrm{E} 1}}>0 \\
& \frac{r_{\mathrm{L} 2}^{*}}{r_{\mathrm{P} 2}^{*}}-\frac{r_{\mathrm{L} 1}^{*}}{r_{\mathrm{P} 1}}>0
\end{aligned}
$$

The expressions (12b) and (12c) correspond to $X=I$ and the expressions (12d) and (12e) to $X=L$ while the expression (12a) holds for both the lumbrical and the 

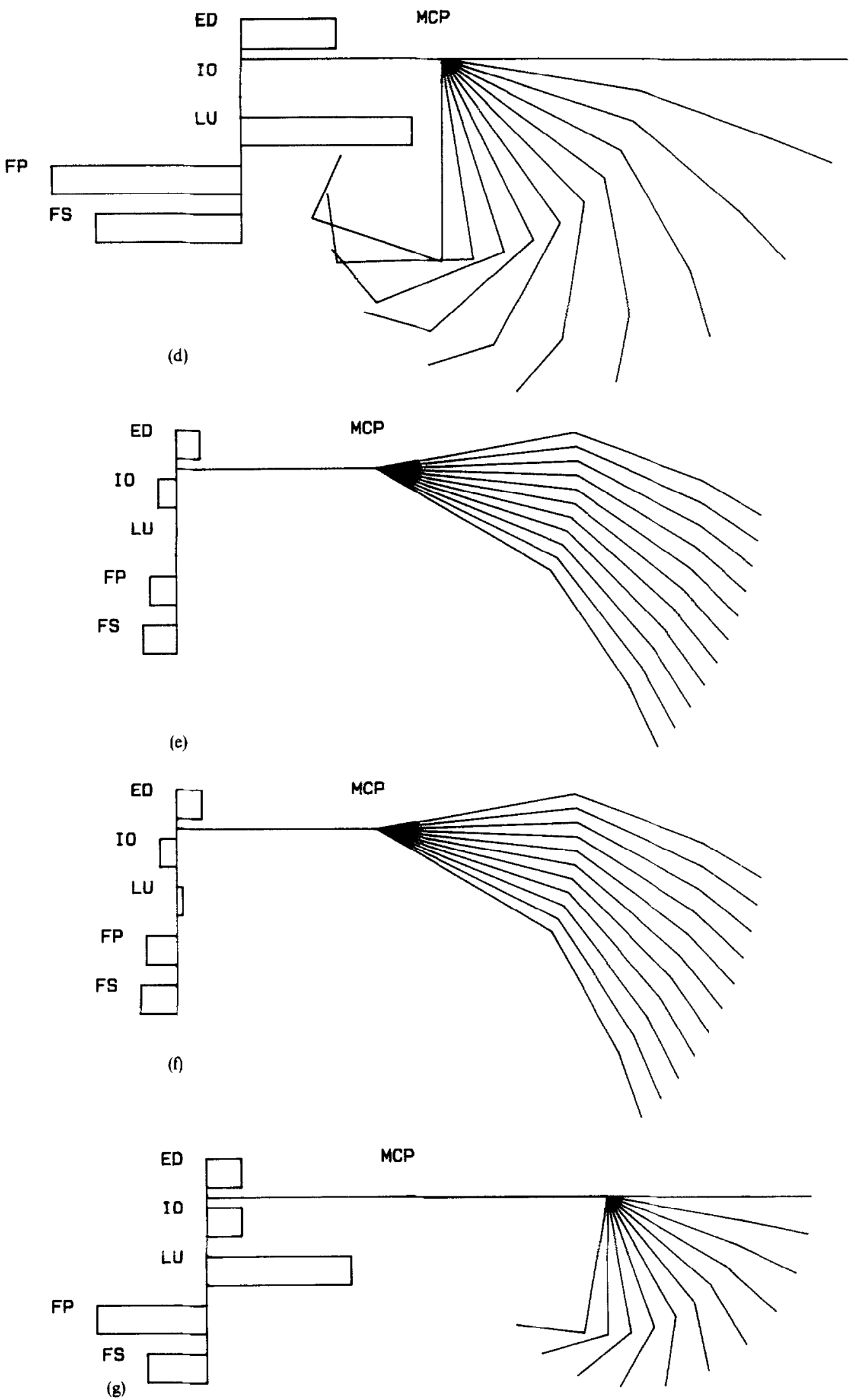

Fig. 4. Finger movements and displacements, calculated from Table. 1 and expression (9): (a) isometric deep flexor; (b) isometric extensor; (c) isometric superficial flexor; (d) isometric interosseus: (e) isometric lumbrical; (f) isometric PIP; (g) isometric MCP. 
interosseus as it contains only the moment arms of extensor and deep flexor (the extrinsics). This condition (12a) is satisfied in the normal finger, as $r_{\mathrm{E} 1}>r_{\mathrm{M} 2}$, and $r_{\mathrm{P} 1}<r_{\mathrm{P} 2}^{*}$. Conditions (12b) and (12c) for the interosseus are satisfied because all terms are positive. Conditions (12d) and (12e) for the lumbrical hold because $r_{\mathrm{L} 2}^{*} \gg\left|r_{\mathrm{L} 1}^{*}\right|$, and $r_{\mathrm{E} 1}>r_{\mathrm{M} 2}$. It thus follows that, when $\sigma_{\mathrm{L}}^{*}, \sigma_{\mathrm{p}}^{*}=0$, the interosseus and lumbrical in the normal finger are mathematically mutually redundant. This was also concluded by Thomas et al. (1968) from a force model

The displacements of lumbrical and interosseus in some basic finger movements. Even though in the two-dimensional finger model with all tendons taut $\left(\sigma_{\mathrm{L}}^{*}, \sigma_{\mathrm{p}}^{*}=0\right)$ the interosseus and lumbrical are mutually redundant, their systemic moment arms greatly differ:

$$
\begin{aligned}
&\left|r_{\mathrm{L} 1}^{*}\right| \ll r_{\mathrm{I} 1}, \\
& r_{\mathrm{L} 2}^{*} \gg r_{\mathrm{M} 2},
\end{aligned}
$$

which implies that the way in which they effectuate their mathematically equivalent functions may also be quite different. This is investigated by calculating the motor displacements in the finger movements generated by keeping alternately one motor or joint isometric [as in Leijnse et al. (1992)].

Finger movement with isometric deep flexor $\left(\mathrm{d} \varepsilon_{\mathrm{p}}=0\right)$. With the deep flexor taut and isometric $\left[\mathrm{d} \varepsilon_{\mathrm{p}}=0, \sigma_{\mathrm{p}}^{*}=0\right.$ in expression (4a)], the feasible joint rotations and the relative displacements of lumbrical and interosseus are (with $r_{\mathrm{L} 1}$ the anatomic moment arm) [Fig. 4(a)]:

$$
\begin{aligned}
\mathrm{d} \theta_{1} & =-\frac{r_{\mathrm{P} 2}^{*}}{r_{\mathrm{P} 1}} \mathrm{~d} \theta_{2}, \\
\mathrm{~d} \varepsilon_{\mathrm{I}} & =\frac{r_{11} r_{\mathrm{P} 2}^{*}+r_{\mathrm{M} 2} r_{\mathrm{P} 1}}{r_{\mathrm{L} 1} r_{\mathrm{P} 2}^{*}+r_{\mathrm{M} 2} r_{\mathrm{P} 1}} \mathrm{~d} \varepsilon_{\mathrm{L}} .
\end{aligned}
$$

Figure 4(a) shows that with isometric deep flexor the MCP flexes and the PIP extends when the interosseus or lumbrical contract. From equation (14) it follows that their displacements differ only to the degree that $r_{L_{1}}>r_{11}$. Physically this is easily understood. With isometric deep flexor, the lumbrical origin is fixed, and can be imagined on the metacarpals, just as the interosseus. As the tendons of the interosseus and lumbrical are almost identical (the only difference being: $\left.r_{L 1}>r_{I 1}\right)$, their displacements are also almost equal.

Isometric extensor $\left(\mathrm{d} \varepsilon_{\mathrm{E}}=0\right)$; isometric superficial flexor $\left(\mathrm{d} \varepsilon_{\mathrm{S}}=0\right)$. With the extensor or the superficial flexor isometric, with all tendon parts taut, the joint rotations [see Fig. 4(b) and 4(c)] are, respectively, given by

$$
\mathrm{d} \theta_{1}=-\frac{r_{\mathrm{E} 2}}{r_{\mathrm{E} 1}} \mathrm{~d} \theta_{2}, \quad \mathrm{~d} \theta_{1}=-\frac{r_{\mathrm{S} 2}}{r_{\mathrm{S} 1}} \mathrm{~d} \theta_{2}
$$

Isometric interosseus $\left(\mathrm{d}_{\varepsilon_{\mathrm{I}}}=0\right)$. With an isometric interosseus, the joint rotations and motor displacements [Fig. 4(d)] are given by

$$
\begin{aligned}
& \mathrm{d} \theta_{1}=\frac{r_{\mathrm{M} 2}}{r_{\mathrm{I} 1}} \mathrm{~d} \theta_{2}, \\
& \mathrm{~d} \varepsilon_{\mathrm{L}}=-\frac{r_{\mathrm{L} 1}^{*} r_{\mathrm{M} 2}+r_{\mathrm{L} 2}^{*} r_{\mathrm{I} 1}}{r_{\mathrm{I} 1}} \mathrm{~d} \theta_{2}, \\
& \mathrm{~d} \varepsilon_{\mathrm{p}}=\frac{r_{\mathrm{P} 1} r_{\mathrm{M} 2}+r_{\mathrm{P} 2}^{*} r_{\mathrm{I} 1}}{r_{\mathrm{I} 1}} \mathrm{~d} \theta_{2}, \\
& \mathrm{~d} \varepsilon_{\mathrm{E}}=-\frac{r_{\mathrm{E} 1} r_{\mathrm{M} 2}+r_{\mathrm{E} 2} r_{\mathrm{I} 1}}{r_{\mathrm{I} 1}} \mathrm{~d} \theta_{2} .
\end{aligned}
$$

From equations (16a) and (4f), it follows that in this movement all joints extend or flex together, as in the opening and closing of the hand (fist). While the displacement of the interosseus is zero, the displacements of all other motors, including the lumbrical, are maximal. Clearly, in this movement the functioning of interosseus and lumbrical is completely different. The interosseus acts as a tenodesis, while the lumbrical maximally contracts or extends.

Isometric lumbrical $\left(\mathrm{d} \varepsilon_{\mathrm{L}}=0\right)$. With the lumbrical isometric and all tendon parts taut, the joint rotations and motor displacements are [Fig. 4(e)]:

$$
\begin{aligned}
\mathrm{d} \theta_{2} & =-\frac{r_{\mathrm{L} 1}^{*}}{r_{\mathrm{L} 2}^{*}} \mathrm{~d} \theta_{1}, \\
\mathrm{~d} \varepsilon_{\mathrm{p}} & =\frac{r_{\mathrm{P} 1} r_{\mathrm{L} 2}^{*}-r_{\mathrm{P} 2} r_{\mathrm{L} 1}^{*}}{r_{\mathrm{L} 2}^{*}} \mathrm{~d} \theta_{1}, \\
\mathrm{~d} \varepsilon_{\mathrm{E}} & =\frac{-r_{\mathrm{E} 1} r_{\mathrm{L} 2}^{*}+r_{\mathrm{M} 2} r_{\mathrm{L} 1}^{*}}{r_{\mathrm{I} 2}^{*}} \mathrm{~d} \theta_{1}, \\
\mathrm{~d} \varepsilon_{1} & =\frac{r_{1} r_{\mathrm{L} 2}^{*}+r_{\mathrm{M} 2} r_{\mathrm{L} 1}^{*}}{r_{\mathrm{L} 2}^{*}} \mathrm{~d} \theta_{1},
\end{aligned}
$$

Figure 4(e) shows that in this movement the PIP is quasi isometric.

Isometric PIP $\left(\mathrm{d} \theta_{2}=0\right)$. The quasi isometry of the PIP in movements with isometric lumbrical is illustrated by the fact that the small approximation of putting the systemic moment arm $r_{L_{1}}^{*}=r_{P_{1}}-r_{L_{1}}$ equal to zero $\left(r_{L_{1}}^{*}=0\right)$ in expression (17) results in a finger movement with a strictly isometric PIP [Fig. 4(f)]:

$$
\begin{aligned}
\mathrm{d} \theta_{2} & =0, \\
\mathrm{~d} \varepsilon_{\mathrm{p}} & =r_{\mathrm{P} 1} \mathrm{~d} \theta_{1}, \\
\mathrm{~d} \varepsilon_{\mathbf{E}} & =-r_{\mathbf{E} 1} \mathrm{~d} \theta_{1}, \\
\mathrm{~d} \varepsilon_{\mathrm{I}} & =r_{\mathrm{I} 1} \mathrm{~d} \theta_{1} .
\end{aligned}
$$

In other words, with $r_{L_{1}}^{*}=0$ and the lumbrical isometric, the distal joints (PIP and DIP) are fixed $\left(\mathrm{d} \theta_{2}=\mathrm{d} \theta_{3}=0\right)$, while the displacements of the other motors move only the MCP. In the Fig. 4(f) the displacement of the lumbrical is as in a finger with isometric PIP and normal $r_{\mathrm{L} 1}^{*}$ value. 
Isometric $M C P\left(\mathrm{~d} \Theta_{1}=0\right)$. The movement of PIP rotation with immobile MCP is presented in Fig. $4(\mathrm{~g})$. Notice that in this movement the displacement of the lumbrical is the largest of all motors.

\section{DISCUSSION}

\section{The lumbrical loop}

The systemic moment arm $r_{\mathbf{L}_{1}}^{*}$ of the lumbrical at the MCP is small. Therefore, the functioning of the lumbrical will not be substantially altered when this moment arm is assumed zero $\left(r_{\mathbf{L}_{1}}^{*}=0\right)$, as in the Fig. $5(a)$ and $5(b)$. The lumbrical origin may then be shifted distal to the MCP without any effect on function, as in the Fig. 5(c) and 5(d) [in Fig. 5(c) a pulley is introduced to keep the tendons on a longitudinal course]. The equivalent representations of these figures in Fig. 5(e) and $5(f)$ clearly illustrate that the lumbrical-deepflexor complex consists of three systemic parts: (i) the lumbrical, (ii) the part $P_{\mathrm{p}}$ of the deep flexor proximal to the lumbrical origin, and (iii) the part $P_{\mathrm{d}}$ of the deep flexor distal to the lumbrical origin. Hereby the lumbrical and the $P_{\mathrm{d}}$ form a closed loop around the distal two joints, which is kept taut by the $P_{\mathrm{p}}$. This construct may be explored as follows.

First, assume the lumbrical to be a rope of fixed length; the MCP immobile (arthrodised); and the L- $P_{d}$ loop slack. When the deep flexor $\left(P_{p}\right)$ then shortens, the attachment of the lumbrical loop displaces proximally, thus removing equal slackness in both sides of the loop until either the lumbrical rope or the $P_{d}$ tautens. As the $P_{p}$ further shortens, the PIP-DIP joints will extend or flex, depending on which part of the loop is taut, until the other part of the loop also tautens. At that point, the PIP-DIP joints become locked, since further shortening of the $P_{p}$ would require them to flex and extend at the same time. This phenomenon can be appreciated from the equivalent model of Fig. 5(d): when the lumbrical and the deep flexor are taut, the equivalent PIP joint is blocked.

When the MCP is now rotated up and down (say, by manually moving the proximal phalanx) while the $\mathbf{P}_{\mathbf{p}}$ is kept taut, the lumbrical loop will remain taut and the distal joints will remain stably fixed, even if the $P_{p}$ elongates or shortens because of its displacement at the MCP. No changes of length within the lumbrical loop occur because this loop does not cross the MCP, which means that the PIP-DIP positions are independent of the MCP movement.

When the MCP is let free, with the $P_{p}$ taut, the extensor $E$ must tauten to control the MCP at the extension side. When the conditions (12) hold, the extensor will not disturb the position of the distal joints, and the lumbrical loop will remain taut.

\section{EPL-MCP rotation}

From the above, it follows that when the lumbrical loop is taut and of constant length, the distal finger is a rigid entity, and the finger is only mobile at the
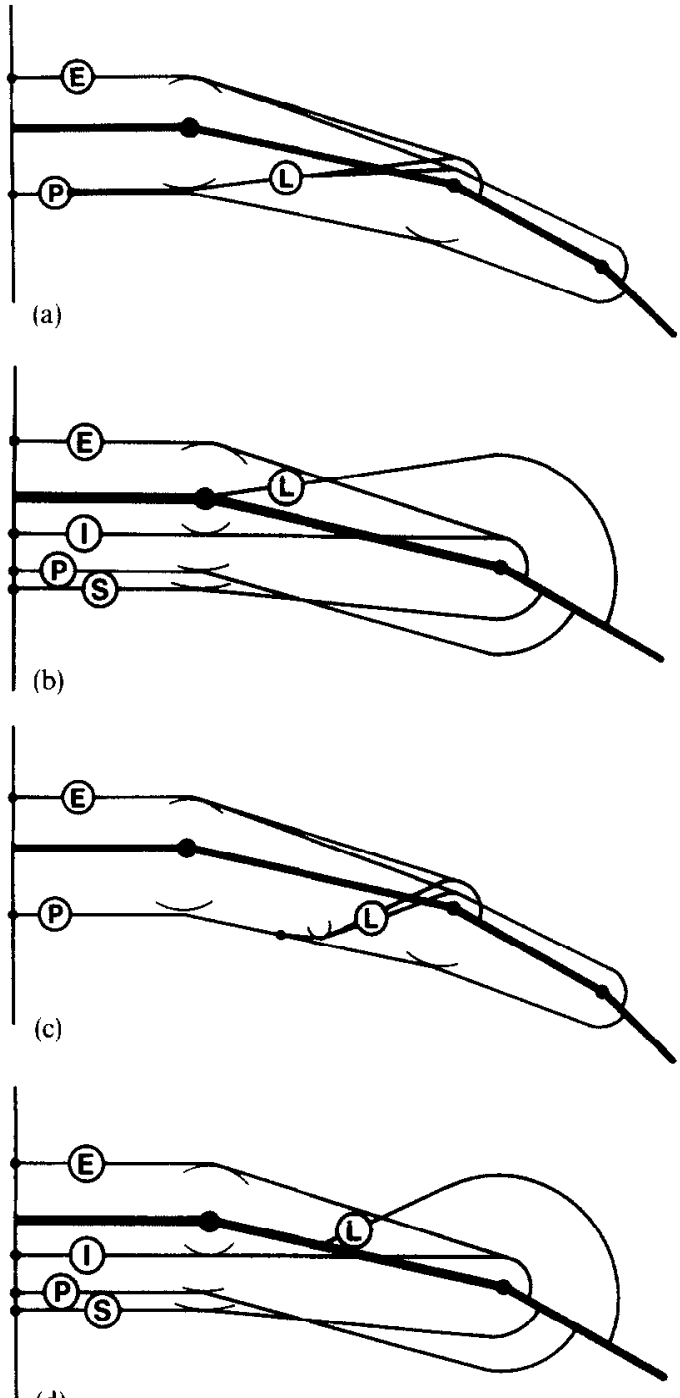

(d)
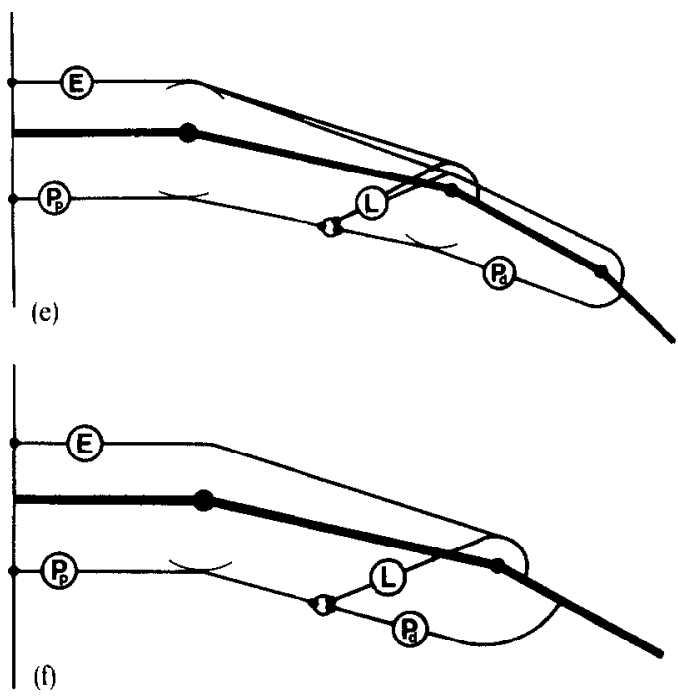

Fig. 5. (a) Finger model with $r_{\mathrm{L} 1}^{*}=0$; (b) bi-articular equivalent model of Fig. 5(a); (c) finger model with lumbrical origine distal to the MCP; (d) bi-articular equivalent model of Fig. 5(c); (e) the lumbrical loop; (f) bi-articular equivalent of Fig. 5(e) (with $r_{P 2}^{*}$ and $r_{L 2}^{*}$ constant). 
MCP. At this joint, the deep flexor and extensor form an antagonistic motor pair which can rotate (oscillate) the entire rigid finger as a simple one-joint-two-tendon unit. Hereby the condition of tautness of the lumbrical loop required to lock the distal joints equals the condition of tautness of the $P_{p}$ required for flexion control of the MCP. The EPL-MCP rotation movement corresponds to the expression (18), and Fig. $4(\mathrm{f})$.

\section{Lumbrical control of the position of the distal joints}

The position of the PIP-DIP joints is determined by the relative lengths of the flexion $\left(P_{d}\right)$ and extension (L) part of the lumbrical loop. To change the PIP-DIP position, it suffices to change the length of one part of the loop relative to the other part. A lengthening of the lumbrical slackens the extension side of the loop, and when the $P_{p}$ shortens to keep the loop taut, the PIP-DIP joints flex until the lumbrical is taut again. Conversely, the shortening of the lumbrical pulls the $P_{p}$ distally and slackens the $P_{d}$, causing the distal joints to extend until the $P_{d}$ is taut again.

The effects of changes in lumbrical length on the MCP position

The lengths of the other bi-articular motors are not independent of the length of the lumbrical. Changes in lumbrical length cause changes in the position of the distal joints, which result in shortages or excesses of length in the motors which cross these joints. Since for proper finger control these motors must remain taut, these excesses or shortages of length must be corrected, and the way in which this is done determines the total finger movement. The first two movements of Fig. 4 may serve as an illustration, when interpreted as resulting from lumbrical shortening. In the first example [Fig. 4(a)], the $P_{p}$ remains isometric, while the extensor lengthens despite excess length produced by the extending distal joints, since its distal displacement at the MCP is greater. In the second movement [Fig. 4(b)], the extensor is isometric, while the $P_{p}$ lengthens to accommodate the shortage of length created by the extending distal joints.

The experiments of Backhouse and Catton, and Ranney et al.

In the experiments of Backhouse and Catton (1954), and Ranney et al. (1987), the lumbrical is made to contract against the real (Backhouse) or simulated (Ranney) elastic forces of the other finger motors. Hereby it was invariably noted that simultaneous MCP flexion, and PIP-DIP extension occurred. These results were cxplaincd in Ranney et al. (1987) and Ranney and Wells (1988) as due to the interaction of the lumbrical with the elastic forces of the bi-articular extrinsic motors (E, P,S) of the finger. To illustrate their argument further, especially the effect of isolated lumbrical contraction on the MCP, the experiments of Ranney et al. (1987) are imaginarily repeated with the model of Fig. 5(d). Notice that in this model the lumbrical has no direct effect on the $\mathrm{MCP}$, as it does not cross this joint.
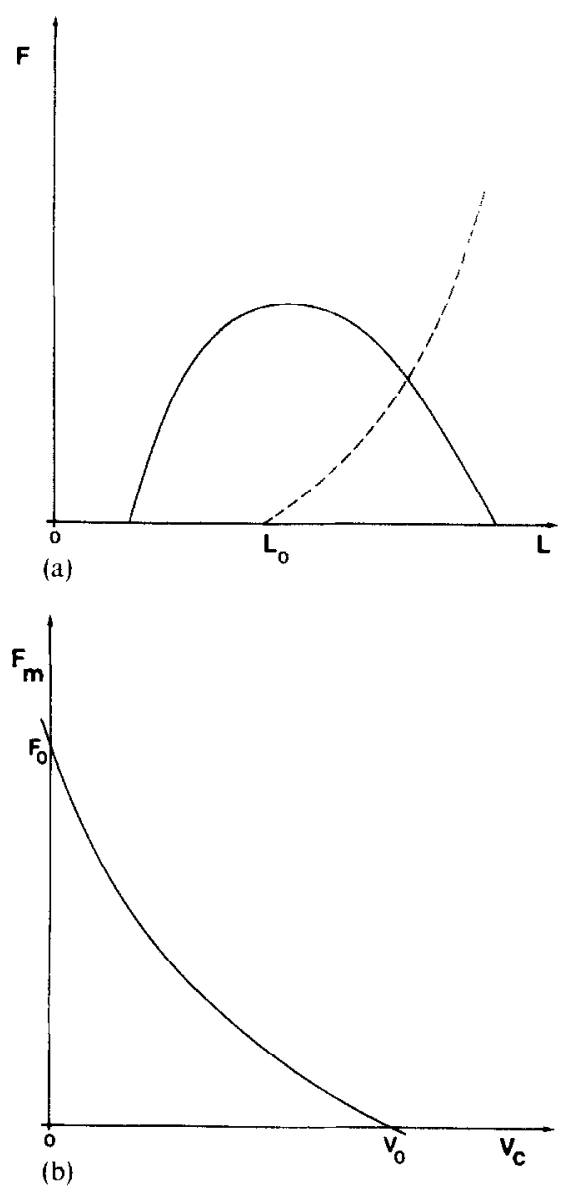

Fig. 6. (a) Elastic force (- - ) and maximal active force (_-)-length relationship of a muscle; (b) maximalforce-contraction-speed $\left(F_{\mathrm{m}}-v_{\mathrm{c}}\right)$ relationship of a muscle.

Assume the finger motors with their normal physiological elastic properties [Fig. 6(a)], and the finger in the resting position. While keeping the MCP externally fixed, let the lumbrical in Fig. 5(d) contract. As described above, this contraction will extend the PIP joint, thus creating an excess of length in the extensor, which leads to a shortening of the extensor and a decrease in its elastic pull. Meanwhile, the deep flexor is stretched by the extending PIP joints, and its elastic pull is increased. The lumbrical contraction thus results in a net flexion moment at the MCP. Therefore, when let free this joint will flex, thereby increasing and decreasing the length (and the elastic pull) of the extensor and the flexor, respectively, until it is balanced again. The resulting movement will be quasi identical to the movements in the experiments of Ranney et al., the only difference resulting from the approximation $r_{L_{1}}^{*}=0$. This shows that even if the lumbrical in Fig. 5(d) has no direct effect on the MCP, its action will flex this joint through the coupling of the MCP-PIP joint rotations by the bi-articular motors. The resulting movement will be some average of the movement with isometric $P$ and isometric $E$ (Fig. 4 (a) and 4(b), respectively), i.e. with flexing MCP and extending PIP, and with both the extensor and deep flexor a bit elongated. 
The mutual redundancy of the lumbrical and interosseus in the Landsmeer model

An illustration to the above discussion is the interpretation of the mutual redundancy of the lumbrical and interosseus within the three-tendon bi-articular model of Spoor and Landsmeer (1976), which focusses on the coupling of the MCP-PIP rotations by the extrinsics (flexor and extensor). The flexor and extensor span both joints as antagonists, and their simultaneous actions result in a collapse (zigzag movement) of the chain. This movement cannot be reversed by these two motors (since they cause it), meaning that for complete control of the chain a third motor is required, which is capable of reversing this zigzag. The conditions (12) may be interpreted from this point of view. Condition (12a) only contains the moment arms of the flexor and extensor, and asserts that the zigzag movement is with extending MCP and flexing PIP. The other expressions (12b) and (12c), or (12d) and (12e) provide the ranges of the moment arms of the lumbrical and interosseus which allow these motors to reverse the zigzag. These expressions allow for a wide variability in the moment arms of the lumbrical and interosseus which still allow effective finger control:

$r_{\mathrm{M} 2}=0$ : mono-articular interosseus,

$r_{\mathrm{I}}=0$ : bi-articular interosseus with zero MCP moment arm.

$r_{\mathrm{L} 1}^{*}=0$ : mono-articular lumbrical, as in Fig. 5(d),

$r_{11}<0$, with $\left|r_{11}\right|<\left(r_{\mathrm{P} 1} / r_{\mathrm{P} 2}^{*}\right) r_{\mathrm{M} 2}$ : interosseus shifted to the extension side of the MCP, etc.

Whatever their appearance, all these variations produce an equal effect: the reversal of the joint rotations as resulting from extrinsic action. A mono-articular interosseus will achieve this by antagonising the extension of the MCP in the zigzag, while a mono-articular lumbrical will do so by opposing the flexion of the PIP. The collapse antagonist may even he a zigzag agonist at one joint, as long as it antagonises the movement of the other joint to a greater extent, as is the case with an interosseus with $r_{11}<0$ (i.e. with a moment arm of extension at the MCP) and $\left|r_{11}\right|<\left(r_{\mathrm{P} 1 / r_{\mathrm{P} 2}}^{*}\right) r_{\mathrm{M} 2}$. With respect to the experiments of Ranney et al. (1987), it follows that in the free moving finger all motors satisfying expression (12) will produce a movement of simultaneous MCP flexion and PIP extension (albeit of different degree) as the inverse of the simultaneous MCP extension and PIP flexion of the extrinsic zigzag, even if e.g. their (systemic) moment arms at the MCP are of extension, as is the case with the lumbrical in the human finger.

\section{The lumbrical as PIP-DIP position proprioceptor}

The role of the lumbrical as a proprioceptor of the position of the distal joints has been pointed out, on the basis of its anatomic position and the extent of its proprioceptic innervation (Rabischong, 1962; Ranney and Wells 1988; Stack, 1962). The above discussion conforms with this view, as (i) the lumbrical length is quasi independent of the MCP position, while (ii) the very large systemic moment arm $r_{\mathrm{L} 2}^{*}$ allows for good conditioned measurement, as small rotations of the PIP-DIP joint mechanism correspond to large lumbrical displacements. The latter point also holds to a somewhat lesser extent for the deep flexor.

\section{Fast movements with isometric lumbrical $\left(\mathrm{d} \varepsilon_{\mathrm{L}}=0\right)$ and isometric $M C P\left(\mathrm{~d} \theta_{1}=0\right)$}

In fast movements, physiological constraints which limit execution speed become active, such as the 'maximal muscle force-contraction speed' $(F-v)$ relationship [Fig. 6(b)]. The lumbrical displacements in movements with large PIP-DIP rotations are considerable, because of the large systemic momentarm $r_{\mathrm{L} 2}^{*}$. Since the lumbrical is also a weak motor, the physiological $(F-v)$ constraint may limit effective lumbrical function when such movements are excuted fastly. In the following two movements are compared from this point of view: (i) the movement with isometric lumbrical, and (ii) the movement with isometric MCP.

Isometric lumbrical [Fig. 4(e)]. In the movement with isometric lumbrical (EPL-MCP rotation) the lumbrical contraction speed is zero, and the physiologically applicable lumbrical force is maximal. The displacements of the other motors serve only to control the MCP trajectory, as the isometric lumbrical loop blocks the distal joints, and the finger functions basically as a mono-articular joint. Such a movement has the aspect of being easily controllable in the fast execution: only one joint trajectory must be effectively controlled, and as none of the motor displacements is large, no motor in particular will be subjected to the $(F-v)$ constraint. With respect to application, such a control strategy seems well apt as a basis for e.g. a fast key stroke in playing of piano.

Isometric MCP [Fig. 4(g)]. In the flexion/extension movement of the distal finger (PIP DIP joints) with isometric $\mathrm{MCP}\left(d \theta_{1}=0\right)$, the lumbrical displacement approaches the physiological maximum, and exceeds that of any other motor [Fig. 4(g)]. Therefore, in the fast execution, the $(F-v)$ constraint should be most active in the lumbrical. This implies that the amplitude of the PIP oscillation, when lumbrical controlled, should decrease with speed, and/or that the interosseus must provide support. It can also be noticed that the isometry of the MCP $\left(d \theta_{1}=0\right)$ is the result of a strict relationship between the simultaneous non-zero displacements of minimally three motors $(\mathbf{E}, \mathbf{P}, \mathbf{I}$ or $\mathrm{L})$. This may indicate that this movement requires a greater coordinative effort than the EPL-MCP rotation, in which the (quasi-) isometry condition $\mathrm{d} \theta_{2}=0$ corresponds to the isometry condition of a single motor: $d \varepsilon_{\mathrm{L}}=0$.

\section{Movements with isometric MCP or PIP in the real finger: some intuitive experimental evidence}

Some direct experimental evidence about the existence of the EPL-MCP rotation strategy in the human finger may be obtained by executing a fast pianistic 'thrill', or a fast tapping with a single finger on the table. The reader may notice that such movement is 
well executable with (quasi) isometric PIP-DIP joints. Hereby the relative 'stiffness' of the immobile distal finger does not appreciably influence the mobility of the MCP [the independence of MCP mobility and lumbrical activity was noted in the EMG studies of Backhouse and Catton (1954) and Long and Brown 1964)]. This movement may be compared with the movement of PIP-DIP flexion/extension with isometric MCP [during this movement, the MCP joint should not be in a position of hyper-extension, as in such position the volar ligaments are taut (functional end-position), and the model (4) does not apply anymore]. When slowly executed, the PIP-DIP flexion/extension movement is unproblematic and well controllable. However, with increasing execution speed, it is generally more difficult to keep the MCP immobile, and/or to maintain a large amplitude of PIP-DIP flexion/extension. On the whole, most subjects tested at our department found that keeping the MCP immobile with fast PIP-DIP flexion/extension movements required greater effort than keeping the distal joints immobile during MCP rotations with isometric PIP DIP joints.

\section{The coupled action of lumbrical and interosseus in the finger with bi-axial MCP joint}

In the real finger, the MCP is bi-axial (flexion/ extension, abduction/adduction), and the moment arm of the lumbrical for the abduction/adduction axis of the MCP is non-zero. This means that the actions of the lumbrical (radial abduction) and the contralateral interosseus (ulnar abduction) are coupled by the abduction-adduction equilibrium condition of the MCP. Therefore, in the real finger the lumbrical and the interossei are not entirely mutually redundant, as any lumbrical action necessarily implies (some) ulnar interosseus activity.

\section{General constraints on fast movements in the hand of the musician}

The above discussion suggests that not all feasible finger movements can be executed fast, because of physiological and coordinative constraints which become active when movements are speeded up. In the real hand, other constraints on movement are possible, such as the limitations on tendon displacements as resulting from anatomic connections between tendons (Leijnse et al., 1992, 1993). These anatomic constraints may further limit the feasible fast movements in the individual hand, the more so since they may cause slack in tendon parts on which systemic lumbrical function depends.

\section{CONCLUSION}

In this paper the functioning of the lumbrical is studied in a kinematic model which describes the feasible movements of the freely moving finger in the sagittal plane. To this end, a standard finger model is represented in equivalent forms from which lumbrical function is visually more clear. From the kinematic equations and the equivalent models. the following statements about lumbrical function can be verified.

The lumbrical-deep-flexor complex consists of the deep flexor proximal to the lumbrical origin in series with two parallel elements: the lumbrical and the deep flexor tendon distal to the lumbrical origin. The functioning of the lumbrical depends critically on the slackness or tautness of these three tendon parts. Hereby the distal deep flexor tendon is systemically taut only when the lateral band of the extensor assembly is also taut. On the basis of tendon slacks. the following functions can be distinguished:

(a) When the part of the deep flexor proximal to the lumbrical origin is slack, the lumbrical loses its fixation, and any effective function.

(b) When the tendon of the deep flexor distal to the lumbrical origin is slack, the lumbrical and the deep flexor motor form a single (digastric) motor, which is systemically equivalent to an interosseus with a slightly greater moment arm at the MCP joint.

(c) With all mentioned tendon parts taut, the following statements hold:

- the lumbrical forms with the distal deep flexor tendon a loop enclosing and able to control the PIP and DIP joints, which coordinate as a mechanism. The length of this loop is quasi independent of the MCP position, and the lumbrical effectively functions as a dedicated controller of the position of the distal two joints. Hereby it has a very large systemic moment arm of extension for the PIP-DIP mechanism.

- in the two-dimensional model, the lumbrical and the interosseus provide mathematically the same function, and are therefore mutually redundant. However, their displacements in movements may be quite different, as e.g. when making a fist: in that case the interosseus is quasi isometric, while the lumbrical elongates maximally.

- the systemic moment arms of the deep flexor and lumbrical for the PIP-DIP-mechanism increase with PIP flexion, because they are function of the variable moment arm of the lateral band at the PIP joint.

- the lumbrical is in an optimal position for proprioceptic feedback on the position of the PIP-DIP joint mechanism because of its large systemic moment arm for this mechanism, and its very small systemic moment arm at the MCP.

- the effect of the lumbrical on the MCP results basically from the coupling of the MCP-PIP joint rotations by the bi-articular finger motors (extrinsics).

- with the PIP/DIP position controlled by the lumbrical loop, the MCP joint (in the sagittal plane) can be controlled by only the extensor and decp flexor.

- with isometric lumbrical, the distal joints are fixed and the finger can be controlled at the MCP by the extensor and deep flexor as a simple onejoint-two-tendon structure. This movement may bc of 
importance as a basis of fast movements in e.g. the musicians' hand.

Acknowledgements-The authors cordially thank J. M. F Landsmeer, C. J. Snijders, J. C. Van der Meulen, S. E. R. Ilovius and G. J. Sonneveld for their valuable contributions.

\section{REFERENCES}

Backhouse, K. M. and Catton, W. T. (1954) An experimenta study of the functions of the lumbrical muscle in the human hand. $J$. Anat. 88, 133-141.

Brand, P. W. (1985) Clinical Mechanics of the Hand, C. V. Mosby, St. Louis, p. 285.

Garcia-Elias, M., An, K. N., Berglund, L., Linscheid, R. L. Cooney W. P. and Chao E. Y. S. (1991) Extensor mechanism of the fingers. I. A quantitative study. J. Hand Surg. 16A, 1130-1136.

Leijnse, J. N. A. L., Bonte, J. E., Landsmeer, J. M. F., Kalker, J. J. and Van Der Meulen, J. C., Snijders, C. J. (1992) Biomechanics of the finger with anatomical restrictions:the significance for the exercising hand of the musician. J. Biomechanics 25, 1253-1264.

Leijnse, J. N. A. L., Snijders, C. J., Bonte, J. E., Landsmeer, J. M. F., Kalker, J. J., Van Der Meulen, J. C., Sonneveld, G. J. and Hovius, S. E. R. (1993) The hand of the musician: the kinematics of the bidigital finger system with anatomical restrictions. J. Biomechanics 26, 1169-1179.
Long, C. (1968) Intrinsic-extrinsic muscle control of the fingers. J. Bone Jt Surg. 50.A, 973-984.

Long, C. and Brown, M. E. (1964) Electromyographic kinesiology of the hand: muscles moving the long finger. $J$. Bone Jt Surg. 46A, 1683-1706.

Parkes, A. (1970) The "lumbrical plus" finger. Hand, 2 (2) 164-165.

Rabischong, P. (1962) L'innervation Proprioceptique des muscles lombricaux de la main chez l'homme. Revue Chir Orthopedique 48, 234-245.

Ranney, D. A., Richard, P. W. and Dowling, J. (1987) Lumbrical function: interaction of lumbrical contraction with the elasticity of the extrinsic finger muscles and its effect on metacarpophalangeal equilibrium. J. Hand Surg. 12A, $566-575$.

Ranney, D. and Wells, R. (1988) Lumbrical muscle function as revealed by a new and physiological approach. Anat Rec. 222, 110-114.

Spoor, C. W. (1983) Balancing a force on the fingertip of a two dimentional finger model without intrinsic muscles. J. Biomechanics 16, 497-504

Spoor, C. W. and Landsmeer, J. M. F. (1976) Analysis of the zigzag movement of the human finger under influence of the extensor digitorum tendon and the deep flexor tendon. J. Biomechanics 9, 561-566.

Stack, G. H. (1962) Muscle function in the fingers, $J$. Bone $J$ Surg. 44 B, 899-909.

Thomas, D. H., Long II, C. and Landsmeer, J. M. F. (1968) Biomechanical considerations of lumbricalis behaviour in the human finger. J. Biomechanics 1, 107-115. 